\title{
IDENTIFICATION OF A NOVEL TTC19 MUTATION IN A PORTUGUESE FAMILY WITH COMPLEX III DEFICIENCY
}

Célia Nogueira ${ }^{1}$, José Barros ${ }^{2}$, Maria José Sá3 ${ }^{3}$ Luísa Azevedo ${ }^{4}$, Filippo M. Santorelli ${ }^{5}$, Laura Vilarinho ${ }^{1}$

${ }^{1}$ National Institute of Health, Genetics Department, INSA, Oporto, Portugal; ${ }^{2}$ Neurology Unit, Hospital S. António, Oporto, Portugal; ${ }^{3}$ Neurology Unit, Hospital S. João,
Oporto, Portugal; ${ }^{4}$ IPATIMUP, Population Genetics, Oporto University, Oporto, Portugal; 5Fundazione Stella Maris, Molecular Medicine \& Neurogenetics, IRCCS, Pisa, Italy.

\section{INTRODUCTION}

Defects of mitochondrial complex III (CIII) are a relatively rare cause of mitochondrial dysfunction. CIII or ubiquinol-cytochrome $c$ reductase is the third component of the mitochondrial respiratory chain and catalyzes the electrons transfer from reduced coenzyme $Q$ to cytochrome $c$ and is composed of 11 subunits; one encoded by mitochondrial DNA (MT-CYB) and the remaining by nuclear genes [1]. BCS1L gene is a CIII assembly factor. Mutations in MT-CYB and BCS1L genes account for the vast majority of mutations leading to CIII deficiency, and are associated with a wide range of neuromuscular disorders [2].

The human tetratricopeptide 19 (TTC19), encodes a poorly understood member of tetratricopeptide repeat domain 19 located on chromosome 17 and appears to be involved in the correct assembly of CIII. Recently, mutations in $\pi$ TC19 have been described in three unrelated Italian kindred in association with a severe neurodegenerative disease [3].

\section{PATIENTS AND METHODS}

Here, we present a consanguineous Portuguese family (Figure 1) where a severe biochemical deficiency of complex III enzyme activity occurred in four siblings in association with neurological manifestations suggestive of cerebellar ataxia combined with relentless psychiatric manifestations.

Sequence analysis of genomic DNA was performed to identify diseasecausing mutations in the $\pi$ T19 gene.

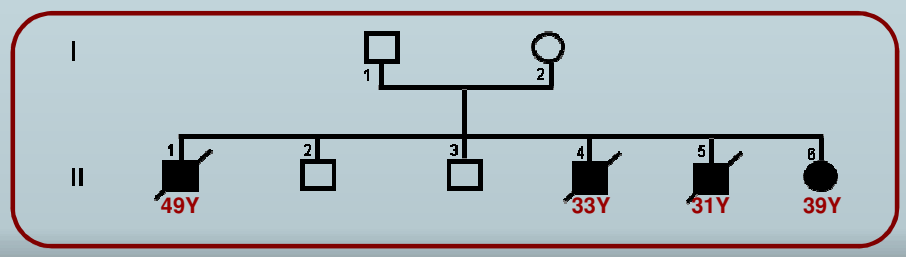

Figure 1 - Portuguese family pedigree.

\section{RESULTS}

We had first detected a biochemically deficient enzyme activity in the family, we had analyzed all structural genes part of CIII as well as $B C S 1 L$. Only the recent description of mutations in $T T C 19$ raised high the suspect of a similar condition in the present family. The novel TTC19 mutation p.A321AfsX8 (c.962_967delTGGC), identified in this family, was homozygous in the four patients, heterozygous in their parents and in two healthy relatives, and it was absent in ethnicallymatched controls (Figure 2).
A

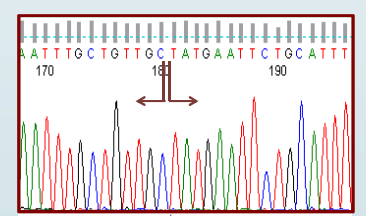

B

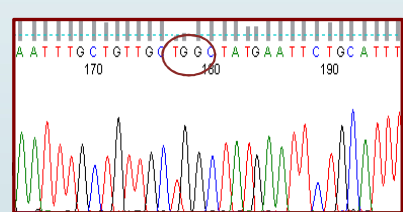

Figure 2 - Sequence analysis of part of TTC19. A) Homozygous patient's sequence with p.A321AfsX8 (c.962_967delTGGC); B) Normal control sequence.

\section{DISCUSSION / CONCLUSION}

In summary, we are describing the 4th family described in the world carrying a novel TTC19 mutation. The identified homozygous mutation, predicts a frameshift and results in a truncated protein by the insertion of a premature stop codon. Variability in age at onset and disease course was presented in the studied family. Our data corroborate the genotype and phenotype variability presented by the affected family members and hopefully will contribute to a deeper understanding of the CIII-related disorders. 